\title{
A etiologia da doença periodontal em obesos e pós-cirurgia bariátrica
}

\author{
The etiology of periodontal disease in obese people and post bariatric surgery \\ Etiología de la enfermedad periodontal en personas obesas y post cirurgia bariátrica
}

Recebido: 21/10/2021 | Revisado: 28/10/2021 | Aceito: 01/11/2021 | Publicado: 02/11/2021

\author{
Amanda Neves Ataide de Oliveira \\ ORCID: https://orcid.org/0000-0002-3444-7009 \\ Centro Universitário de Patos de Minas, Brasil \\ E-mail: amandaataide@unipam.edu.br \\ Ivânia Aparecida Pimenta Santos Silva \\ ORCID: https://orcid.org/0000-0001-7012-8497 \\ Centro Universitário de Patos de Minas, Brasil \\ E-mail: ivaniapimenta@unipam.edu.br \\ Daniella Cristina Borges \\ ORCID: https://orcid.org/0000-0002-5424-5367 \\ Centro Universitário de Patos de Minas, Brasil \\ E-mail: daniellacbroges@unipam.edu.br \\ Leonardo Bíscaro Pereira \\ ORCID: https://orcid.org/0000-0001-9790-9082 \\ Centro Universitário de Patos de Minas, Brasil \\ E-mail: leonardobiscaro@unipam.edu.br \\ Gustavo Augusto Santos \\ ORCID: https://orcid.org/0000-0001-8113-7516 \\ Hospital das Clinicas da Universidade Federal de Minas Gerais, Brasil \\ E-mail: guaugustto@yahoo.com.br \\ Rodrigo Soares de Andrade \\ ORCID: https://orcid.org/0000-0001-6114-0929 \\ Centro Universitário de Patos de Minas, Brasil \\ E-mail: rodrigosa@unipam.edu.br
}

\begin{abstract}
Resumo
A Doença periodontal (DP) é uma inflamação que afeta as estruturas de proteção, suporte ou sustentação de um dente, e que pode estar fortemente associada a obesidade e a pacientes submetidos a cirurgia bariátrica. Esses pacientes fazem parte dos grupos suscetíveis ao desenvolvimento desse tipo de doença, sendo assim considerados fatores de risco. O objetivo desse artigo é abordar a associação da doença periodontal em obesos e em indivíduos submetidos a cirurgia bariátrica, suas causas e como essa doença é manifestada nesses dois grandes grupos. O presente trabalho trata-se de uma revisão integrativa, e foram pesquisados artigos dos últimos 10 anos, relacionados ao assunto nas bases de dados PubMed, com as palavras-chave: "periodontal disease", "obesity", "bariatric surgery". Foram selecionados 17 artigos a partir das buscas e com a aplicação dos critérios de inclusão e exclusão. Diante do exposto, nota-se a necessidade da abordagem de um cirurgião dentista para realizar a avaliação periodontal, prevenção, tratamento e controle tanto no pré, trans e pós-operatório. A equipe que cuida de pacientes submetidos a cirurgia de emagrecimento deve ser multidisciplinar para que esses cuidados sejam feitos de maneira adequada e completa, levando em consideração a importância da manutenção da saúde bucal durante todo o processo de emagrecimento e reestabelecimento da saúde sistêmica desses pacientes.
\end{abstract}

Palavras-chave: Doença periodontal; Obesidade; Cirurgia bariátrica.

\begin{abstract}
Periodontal disease (PD) is an inflammation that affects the structures that protect, support or sustain a tooth, and can be strongly associated with obesity and patients undergoing bariatric surgery. These patients are part of the susceptible groups to the development of this type of disease, and are therefore considered risk factors. The aim of this article is to address the association of periodontal disease in obese patients and in individuals undergoing bariatric surgery, its causes and how this disease is manifested in these two major groups. This is an integrative review, and articles from the last 10 years related to the subject were searched in PubMed databases, with the keywords: "periodontal disease", "obesity", "bariatric surgery". Seventeen articles were selected from the searches and by applying the inclusion and exclusion criteria. In view of the above, it is noted the need for the approach of a dental surgeon to perform periodontal assessment, prevention, treatment and control both preoperatively, transoperatively and postoperatively. The team that cares for patients undergoing weight loss surgery should be multidisciplinary so
\end{abstract}


that such care is done properly and completely, taking into account the importance of maintaining oral health throughout the weight loss process and re-establishing the systemic health of these patients.

Keywords: Periodontal Disease; Obesity; Bariatric Surgery.

\begin{abstract}
Resumen
La enfermedad periodontal (EP) es una inflamación que afecta a las estructuras que protegen, soportan o sostienen un diente, y puede estar muy asociada a la obesidad y a los pacientes sometidos a cirugía bariátrica. Estos pacientes forman parte de los grupos susceptibles al desarrollo de este tipo de enfermedad, por lo que se consideran factores de riesgo. El objetivo de este artículo es abordar la asociación de la enfermedad periodontal en pacientes obesos y en individuos sometidos a cirugía bariátrica, sus causas y cómo se manifiesta esta enfermedad en estos dos grandes grupos. Se trata de una revisión integradora, y se buscaron artículos de los últimos 10 años sobre el tema en las bases de datos de PubMed utilizando las palabras clave: "periodontal disease", "obesity", "bariatric surgery". Se seleccionaron 17 artículos a partir de la búsqueda y la aplicación de los criterios de inclusión y exclusión. Por todo lo anterior, se constata la necesidad del abordaje de un cirujano dentista para realizar la evaluación, prevención, tratamiento y control periodontal tanto en el preoperatorio como en el transoperatorio y el postoperatorio. El equipo que atiende a los pacientes sometidos a cirugía de pérdida de peso debe ser multidisciplinar para que dicha atención se realice de forma adecuada y completa, teniendo en cuenta la importancia de mantener la salud bucodental durante todo el proceso de pérdida de peso y el restablecimiento de la salud sistémica de estos pacientes.
\end{abstract}

Palabras clave: Enfermedad periodontal; Obesidad; Cirugía bariátrica.

\title{
1. Introdução
}

A doença periodontal (DP) é uma inflamação que afeta as estruturas de proteção, suporte ou sustentação de um dente, sendo elas, a gengiva o osso e o ligamento periodontal, o que pode levar a perda dos dentes e contribuir para complicações sistêmicas (Nascimento et al., 2017). A iniciação e propagação da doença periodontal é através do acumulo da placa bacteriana, que estimula as defesas imunológicas do hospedeiro, levando à inflamação e doença. (Kinane; Stathopoulou; \& Papapanou, 2017).

Quando a DP já está mais avançada, é classificada com base no Índice Periodontal Comunitário, que tem pontuação 3 (bolsa periodontal $=4-5 \mathrm{~mm}$ ) e pontuação 4 (bolsa periodontal $\geq 6 \mathrm{~mm}$ ), essas medidas são obtidas por meio do exame clinico utilizando a sonda periodontal (Teixeira et al., 2020). As condições sistêmicas como diabetes, hipertensão e obesidade podem impactar na resposta imune do hospedeiro, assim definindo a progressão e estabelecimento dessa inflamação (Nascimento et al., 2017).

Os agentes patogênicos Aggregatibacter actinomycetemcomitans, Porphyromonas gingivalis, Treponema denticola e Tannerella forsythia são específicos que compõem a periodontite, sendo o $P$. gingivalis, o candidato patogênico mais característico, responsável pela destruição do tecido periodontal. O P. gingivalis é considerado a bactéria principal que determina a virulência de um microbiota oral na periodontite (Yamashita; \& Takeshita, 2017).

A Obesidade $(\mathrm{OB})$ é definida como uma patologia multifatorial, crônica, associada a múltiplas doenças, sendo a desordem nutricional um desencadeador importante no aumento do peso corporal, obtendo um tratamento difícil (Villar et al., 2017). Fatores genéticos, sociais, biológicos, nutricionais e comportamentais podem relacionar com a etiologia da obesidade, a falta de equilíbrio entre o consumo alimentar (calorias) e a inatividade física causa acúmulo excessivo de gordura resultando em obesidade e consequentemente problemas de saúde conhecidos como comorbidades (Prado et al., 2020).

A prevalência da OB, já atingiu cerca de 600 milhões de indivíduos, a OMS estima que 1,9 bilhões de adultos estão acima do peso. Os obesos e tabagistas fazem parte dos grupos suscetíveis a apresentarem a doença periodontal, sendo assim considerados fatores de risco (Franco et al., 2020). Segundo a Organização Mundial da Saúde (OMS), a obesidade é determinada por meio do Índice de massa corporal (IMC). O IMC de uma pessoa é obtido dividindo o seu peso (em quilogramas) pelo quadrado da sua altura (em metros), sendo diagnosticado obeso o indivíduo com IMC maior que $\geq 30 \mathrm{~kg} / \mathrm{m}^{2}$ (Castaños; \& Malatto, 2020). O controle e redução da obesidade podem ser feitos através da cirurgia bariátrica, que irá induz o indivíduo a ter um menor consumo de alimentos e uma restrição na absorção (Moura-Grec et al., 2012). 
Apesar de não ser muito explorada a associação da doença periodontal com pacientes obesos e pós-operatórios de cirurgia bariátrica, é de suma importância compreender sua etiologia, sua manifestação e o porquê afeta com maior frequência esses grupos. O presente trabalho tem como objetivo, entender as causas da doença periodontal nesses pacientes, apresentando uma abordagem multidisciplinar que atenda às necessidades do tratamento.

\section{Metodologia}

\subsection{Tipo de estudo}

O presente trabalho trata-se de um estudo do tipo bibliográfico, descritivo-explicativo, com análise integrativa e qualitativa de caráter básico (Estrela, C. 2018).

\subsection{Estratégias de Pesquisa}

Para o desenvolvimento desse estudo foi realizado pesquisas nas base de dados eletrônica PubMed. Para buscar o material foram usadas as seguintes palavras chave: "Doença periodontal", "obesidade", "sobrepeso", "periodontite", "gengivite", "cirurgia bariátrica" e no inglês: "periodontal disease", "obesity" e "sugery bariatric" foram associados por meio do operador booleano "e" e seu correspondente em inglês. A partir da leitura dos artigos encontrados primariamente, foi selecionado os que têm relação com o objetivo, a partir do título, inicialmente, seguida da leitura do resumo e os selecionados foram lidos na integra para pesquisa do conteúdo.

\subsection{Critérios de Inclusão e Exclusão}

Como critérios de inclusão foram: Artigos na língua inglêsa, publicados nos últimos 10 anos dos anos de 2011 a 2021, artigos na integra. Como exclusão foram: artigos em outras línguas a não ser a citada anteriormente, mais de 10 anos de publicação, artigos que não foram possíveis o acesso do texto completo, trabalhos de conclusão de curso, livros e opiniões de especialista.

\section{Resultados}

A pesquisa retornou com 27 artigos após a aplicação dos filtros e utilização de todas as palavras chaves. Após uma análise crítica dos artigos encontrados, foram selecionados os que tinham relação com o objetivo, a partir dos títulos, seguido da leitura dos resumos. Restaram então, 17 referências lidas na íntegra, relacionadas ao tema que atenderam aos critérios de seleção estabelecidos, assim como é mostrado no fluxograma (Figura 1) e na tabela (Tabela 1). Todos os artigos citados e selecionados tiveram em sua metodologia e discussão o foco em evidenciar a importância da doença periodontal em obesos e pós-bariátricos. 
Figura 1 - Fluxograma do processo de seleção dos estudos primários.

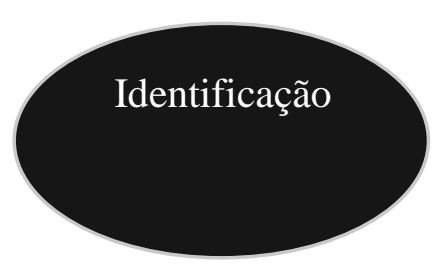

Registro identificado no banco de dados de busca (Pubmed -

Periodontal disease and obesity $n=696$

Periodontal disease and bariatric surgery $\mathrm{n}=28$


Filtro: 10 anos

Textos completos

leitura dos resumos $n=3$

\section{Artigo em texto completo avaliados \\ para elegibilidade $n=17$}

Inclusão

Estudos incluídos em síntese qualitativa $n=17$

Fonte: Autores. 
Tabela 1 - Artigos utilizados na revisão integrativa sobre a prevalência da doença periodontal em obesos e após cirurgia bariátrica.

\begin{tabular}{lll}
\hline $\begin{array}{c}\text { Autor et al. e } \\
\text { ano de } \\
\text { publicação do } \\
\text { artigo }\end{array}$ & Periódico & \multicolumn{1}{c}{ Objetivo } \\
\hline $\begin{array}{l}\text { Pataro et al., } \\
2012\end{array}$ & J.Periodontol & $\begin{array}{l}\text { Determinar a } \\
\text { associação entre o } \\
\text { estado periodontal e } \\
\text { o excesso de peso / } \\
\text { obesidade em } \\
\text { populações pré e } \\
\text { pós-operatórias de } \\
\text { cirurgia no Brasil. }\end{array}$ \\
&
\end{tabular}

$\begin{array}{lllrl}\begin{array}{l}\text { Moura- Grec } \\ \text { et al., 2012 }\end{array} & \text { Arq Bras Cir } & \text { Identificar } & \text { as } & \text { Revisão } \\ & \text { Dig } & \text { consequências da } & \text { sistemática } \\ & \text { cirurgia bariátrica e } & \text { da literatura. } \\ & & \text { suas repercussões na } & \\ & & \text { saúde bucal. }\end{array}$

$\begin{array}{lllr}\text { Levine. } 2012 \quad \text { Br Dent J } & \text { Identificar as } \\ & \text { manifestações das } \\ & \text { doenças bucais em } \\ & \text { obesos. }\end{array}$

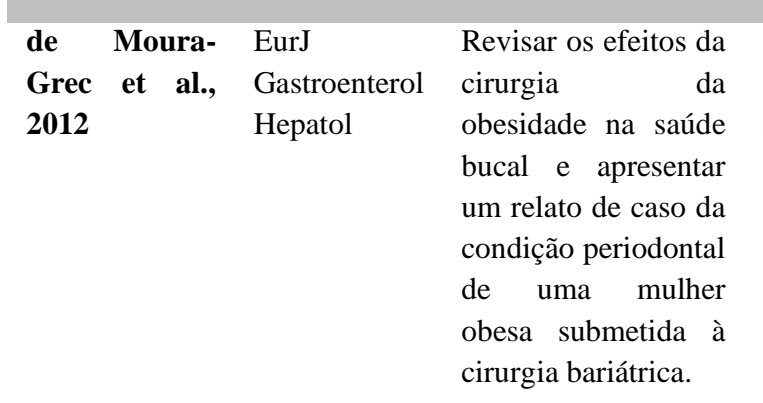

$\begin{array}{lllr}\text { Lakkis et al., } & \mathrm{J} & \text { Avaliar se a perda de } \\ \mathbf{2 0 1 2} & \text { Periodontol } & \text { peso significativa } \\ & \text { melhoraria } & \text { a } \\ & \text { resposta à } & \text { terapia } \\ & \text { periodontal } & \text { não } \\ & \text { cirúrgica } & \text { em } \\ & \text { pacientes obesos. }\end{array}$

\section{Marsicano et Eur J Dent.} al., 2012
O objetivo deste estudo foi identificar a prevalência de cárie dentária, doenças periodontais e desgaste dentário em pacientes bariátricos e relacionar as condições de saúde
Metodologia $\quad$ Resultados

Estudo de Considerando o momento da cirurgia bariátrica, a amostra foi dividida caso controle em três grupos: PRÉ-OP (pré-operatório, $\mathrm{n}=133$ ), POS-OP1 (pósoperatório $\leq 6$ meses, $\mathrm{n}=72$ ) e POS-OP2 (pós-cirurgia $>6$ meses, $\mathrm{n}=$ 140). Independentemente do tipo de cirurgia (PRÉ-OP, POS-OP1, POSOP2), a prevalência de periodontite mostrou-se elevada $(81,45 \%)$. Houve uma diferença estatisticamente significativa na prevalência de periodontite entre os grupos PRE-OP, POS-OP1 e POSOP2 (P = 0,040). No modelo de regressão de Poisson, após o ajuste para variáveis biológicas, demográficas e de risco comportamentais, apenas sangramento na sondagem permaneceu significativamente associado à prevalência de periodontite $(\mathrm{P}<0,001)$.

Algumas complicações da cirurgia são o refluxo crônico e a deficiência nutricional, que podem trazer repercussões na cavidade oral, como erosão dentária, perda óssea e cárie dentária. Por outro lado, também há impactos positivos, como diminuição do diabetes, apneia e melhora da autoestima, que tornam os pacientes menos vulneráveis a complicações de saúde bucal, como xerostomia e doença periodontal.

Pesquisa A pesquisa atual não estabeleceu uma associação clara entre o excesso de adiposidade e a prevalência de cárie, no entanto, há evidências de uma maior prevalência de doença periodontal crônica em populações obesas. Há evidências de que isso não seja mediado apenas pelo diabetes, mas pela secreção pelo tecido adiposo de mediadores químicos da inflamação, incluindo citocinas e hormônios, que podem modificar a resposta dos tecidos periodontais ao meio bucal.

Revisão da Uma busca de estudos relacionados à cirurgia bariátrica e saúde bucal literatura e foi feita no PubMed. Para relato de caso, o IMC, o fluxo salivar e a relato de condição periodontal foram avaliados em três períodos diferentes. Os caso problemas de saúde bucal encontrados foram desgaste dentário, doenças periodontais, xerostomia e cárie dentária. $\mathrm{O}$ paciente, avaliado antes da cirurgia bariátrica e acompanhado por 2 anos, perdeu $40,4 \mathrm{~kg}$, apresentava glicemia normal e hipertensão controlada sem medicação. $\mathrm{O}$ fluxo salivar manteve-se de igual valor no primeiro ano, houve aumento da profundidade média de sondagem e havia baixa densidade óssea nesses locais.

Estudo A média de idade dos participantes do estudo foi de $47,1 \pm 11,5$ anos, e Piloto $\quad 36,7 \%$ dos participantes eram do sexo masculino. Houve uma melhora estatisticamente significativa após a terapia periodontal no BS em comparação com o grupo de obesos ( $\mathrm{P}<0,05)$. O PD teve uma redução média de $0,45 \mathrm{~mm}$ no grupo BS contra $0,28 \mathrm{~mm}$ no grupo controle. A redução no CAL foi de $0,44 \mathrm{~mm}$ versus $0,30 \mathrm{~mm}$, a porcentagem de locais BOP foi de $16 \%$ versus $15 \%$ e GI foi 1,03 versus 0,52 nos grupos BS e controle, respectivamente.

Estudo $\quad \mathrm{O}$ índice CPOD foi de $16,11 \pm 5,19$ no grupo cirúrgico e 16,06 $\pm 6,29$ prospectivo no grupo controle $(\mathrm{P}>0,05)$. O IPC médio foi de $3,05 \pm 0,84$ para o grupo operado e 2,66 $\pm 1,25$ para os pacientes obesos, sem diferença significativa entre eles ( $\mathrm{P}>0,05)$. Houve diferença estatisticamente significativa entre os grupos para a presença de bolsas periodontais $(\mathrm{P}=$ $0,021)$. Todos os pacientes apresentaram algum grau de desgaste dentário, porém, sem diferença significativa entre os dois grupos $(\mathrm{P}=$ 0,82). Os valores médios do fluxo salivar do grupo cirúrgico e do grupo controle foram de $0,64 \pm 0,46 \mathrm{~mL} / \min$ e $0,66 \pm 0,49 \mathrm{~mL} / \mathrm{min}$, respectivamente. Não houve diferença significativa no fluxo de saliva e 
bucal com o fluxo salivar.

$\begin{array}{lll}\text { de } & \text { Moura- Int Dent J } & \text { Avaliamos as } \\ \text { Grec et al., } & \text { condições de saúde } \\ \text { bucal antes e após a } \\ \text { cirurgia bariátrica. }\end{array}$

\section{Pataro et al.}

2016

J App
Sci.

Este

transversal

estudo

comparou

frequência de

periodontopatógenos

orais e H. pylori na

boca e no estômago

de indivíduos obesos

com ou sem

periodontite

submetidos

cirurgia bariátrica.

$\begin{array}{lll}\text { Sales-Peres et } & \text { Surg Obes } & \text { Explorar se a perda } \\ \text { al., 2017 } & \text { Relat Dis } & \text { de peso após a } \\ & & \text { cirurgia bariátrica } \\ & & \text { foi associada a } \\ \text { mudanças } & \text { nas } \\ \text { medidas } & \\ & & \text { periodontais a } \\ & & \text { longo de } 12 \text { meses. }\end{array}$

Bastos et al., J Am Dent Estimular

2018 Assoc. prevalência de

fatores de risco para

cárie, erosão

dentária e doença

periodontal em um

grupo de pacientes

obesos

encaminhados para

cirurgia bariátrica.

$\begin{array}{llll}\begin{array}{l}\text { Fontanille } \\ \text { al., et } 2018\end{array} & \begin{array}{l}\text { Surg Obes } \\ \text { Relat Dis }\end{array} & \begin{array}{l}\text { Revisar e analisar os } \\ \text { parâmetros clínicos } \\ \text { periodontais após } \\ \text { cirurgia bariátrica. }\end{array}\end{array}$

Maria de Surg Obes Investigar o efeito

Souza et al., Relat Dis

2018 da cirurgia bariátrica

no estado

periodontal por meio

de uma revisão

sistemática.

todas as condições orais analisadas $(\mathrm{P}>0,05)$.

Estudo de O IMC médio diminuiu de 49,31 $\pm 8,76$ para 35,52 $\pm 8,12 \mathrm{~kg} / \mathrm{m}$ (2) em coorte 6 meses após a cirurgia $(P<0,000)$. Antes da cirurgia, 67\% dos pacientes apresentavam níveis elevados de PCR e níveis de glicose no sangue 38\% mais elevados e após a cirurgia houve reduções significativas desses níveis $(\mathrm{P}<0,001)$. O fluxo salivar variou de $0,84 \mathrm{a}$ $0,95 \mathrm{ml} / \mathrm{min}$. Houve aumento da prevalência de bolsas periodontais $(\mathrm{P}$ $=0,022)$ e a profundidade média das bolsas aumentou para cerca de 0,5 $\mathrm{mm}(\mathrm{P}<0,001)$. A porcentagem de superfícies com desgaste dentário na dentina foi significativamente maior após a cirurgia bariátrica $(\mathrm{P}=$ $0,002)$, enquanto o desgaste dentário no esmalte diminuiu $(P=0,019)$.

à

Estudo

transversal a

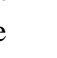

o

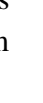

Estudo

Prospectivo A análise estatística demonstrou que os periodontopatógenos foram altamente frequentes na boca (até 91,4\%). No grupo tratado com cirurgia bariátrica, por via oral, $\mathrm{P}$. gingivalis, T. denticola e T. forsythia foram mais frequentes na periodontite, enquanto $C$. rectus foi mais frequente nos indivíduos sem periodontite. As biópsias estomacais também revelaram a alta frequência de cinco espécies orais em ambos os grupos de candidatos à cirurgia bariátrica $(91,6 \%)$ e bariátrico $(83,3 \%)$. H. pylori foi freqüentemente detectado na boca $(50,0 \%)$ e estômago (83,3\%). No estômago, espécies orais e H. pylori apareceram em menor frequência no grupo bariátrico.

$\mathrm{O}$ IMC não foi significativamente relacionado à proporção de locais com BOP no início do estudo, mas foi negativamente associado à taxa de mudança na proporção de locais com BOP. Quanto maior a perda de IMC, maior a proporção de locais com BOP, principalmente 6 meses após a cirurgia. No entanto, o IMC não foi associado à profundidade da bolsa de sondagem da linha de base e perda de inserção clínica ou taxas de alterações nesses resultados periodontais.

Estudo

prospectivo

Os autores avaliaram um total de 255 participantes; $200(78,4 \%)$ eram mulheres, a mediana (intervalo interquartil) de idade era 36,0 (30,0$43,0)$ anos e o índice de massa corporal mediano (intervalo interquartil) era 39,7 (37,6-43,5) quilogramas por metro quadrado. Os autores observaram alta prevalência de fatores de risco para erosão dentária; 78,4\% dos participantes consumiam alimentos ácidos diariamente e 92,2\% consumiam bebidas ácidas diariamente. Com relação aos fatores de risco para cárie, $38,5 \%$ dos participantes relataram ingestão elevada de sacarose e 59,6\% preferiram bebidas doces e alimentos com sacarose. Quase $13 \%$ dos participantes relataram ter ou ter tido mobilidade dentária e $59,6 \%$ relataram ter ou ter sangrado gengival, sugerindo história de doença periodontal.

Revisão de Após uma triagem completa de 651 estudos, 10 estudos foram literatura selecionados por 2 revisores independentes. Quatro $(n=250)$ e 3 sistemática estudos $(n=191)$ foram incluídos na meta-análise em 6 e 12 meses de com meta- acompanhamento, respectivamente. No acompanhamento de 6 meses, analise foram observados aumento da inflamação periodontal $(P=0,03)$ e destruição periodontal. No entanto, 12 meses após o início do estudo, a diferença entre os pacientes bariátricos e o controle não era mais significativa.

Revisão A estratégia de busca resultou em 1878 artigos. Após o processo de sistemática e seleção, nove estudos foram incluídos na análise qualitativa e cinco na meta-analise metanálise. Três estudos transversais mostraram risco de pontuação de viés variando de 5 a 6 estrelas, e estudos de coorte pontuaram de 6 a 9 estrelas de 9 estrelas possíveis na escala Newcastle-Ottawa. A análise quantitativa mostrou que o nível de inserção clínica (MD: 0,07; IC95\% 0,17 a 0,31), índice gengival (MD: -0,28; IC95\% -1,68 a 1,11), 


\begin{tabular}{|c|c|c|c|c|}
\hline $\begin{array}{l}\text { Dos santos et } \\
\text { al., } 2019\end{array}$ & $\begin{array}{l}\text { Surg Obes } \\
\text { Relat Dis }\end{array}$ & $\begin{array}{l}\text { Avaliar a influência } \\
\text { da cirurgia bariátrica } \\
\text { nas condições } \\
\text { clínicas periodontais } \\
\text { de pacientes com } \\
\text { obesidade. }\end{array}$ & $\begin{array}{l}\text { Revisão } \\
\text { sistemática e } \\
\text { meta-analise }\end{array}$ & $\begin{array}{l}\text { A meta-análise foi baseada no método de Mantel-Haenszel e variância } \\
\text { inversa. A análise quantitativa não revelou diferenças estatisticamente } \\
\text { significativas com relação ao sangramento na sondagem }(\mathrm{P}=0,9 \text {; desvio } \\
\text { médio: }-, 70 \text {; intervalo de confiança }=-11,43 \text { a } 10,04) \text { ou profundidade } \\
\text { da bolsa de sondagem }(\mathrm{P}=0,41 \text {; desvio médio: }-.46 \text {; intervalo de } \\
\text { confiança }=-1,55 \text { a } 0,63) \text { antes e depois da intervenção. A perda de } \\
\text { inserção clínica mostrou uma diferença estatisticamente significativa (P } \\
=0,0002 \text {; desvio médio: } 0,18 \text {; intervalo de confiança }=0,07-0,30) \text {. }\end{array}$ \\
\hline $\begin{array}{l}\text { Schwenger et } \\
\text { al., } 2020\end{array}$ & $\begin{array}{l}\text { Surg Obes } \\
\text { Relat Dis }\end{array}$ & $\begin{array}{lr}\text { Avaliar } & \text { a } \\
\text { prevalência } & \text { de } \\
\text { hipossalivação e } & \text { OI } \\
\text { em indivíduos } \\
\text { submetidos ao } \\
\text { bypass gástrico em } \\
\text { Y-de-Roux (BGYR) } \\
\text { e determinar a } \\
\text { relação } \\
\text { parâmetros com } \\
\text { metabólicos } \\
\text { antropométricos } \\
\text { antes e após BGYR }\end{array}$ & $\begin{array}{l}\text { Estudo } \\
\text { prospectivo }\end{array}$ & $\begin{array}{l}\text { Cinquenta e nove pacientes foram incluídos, com } 29 \text { completando este } \\
\text { estudo. No início do estudo, a mediana de idade era de } 47 \text { anos e o } \\
\text { índice de massa corporal de } 46,5 \mathrm{~kg} / \mathrm{m} 2,52(88,1 \%) \text { eram mulheres e } \\
14 \text { indivíduos }(23,7 \%) \text { tinham diabetes tipo } 2 ; 54,2 \%(\mathrm{n}=32) \text { dos } \\
\text { pacientes apresentaram hipossalivação e } 13,6 \%(\mathrm{n}=8) \text { apresentaram } \\
\text { contagem elevada de neutrófilos. Os pacientes com hipossalivação } \\
\text { apresentaram glicose em jejum significativamente maior }(5,7 \mathrm{mmol} / \mathrm{L}) \\
\text { em comparação com aqueles sem hipossalivação }(5,2 \mathrm{mmol} / \mathrm{L}) \text {, mas } \\
\text { nenhuma diferença foi encontrada entre a contagem de neutrófilos alta e } \\
\text { baixa. Aos } 6 \text { meses após o RYGB, todas as variáveis, exceto a contagem } \\
\text { de neutrófilos orais, melhoraram significativamente. A hipossalivação } \\
\text { persistiu em } 7(24 \%) \text { indivíduos. }\end{array}$ \\
\hline $\begin{array}{l}\text { Čolak et al., } \\
2021\end{array}$ & $\begin{array}{l}\text { Arch } \\
\text { Sci. }\end{array}$ & $\begin{array}{l}\text { Nosso objetivo foi } \\
\text { determinar se a } \\
\text { saúde periodontal se } \\
\text { deteriora após a } \\
\text { cirurgia bariátrica } \\
\text { (CB). }\end{array}$ & $\begin{array}{l}\text { Revisão } \\
\text { sistemática e } \\
\text { meta-analise }\end{array}$ & $\begin{array}{l}\text { Os resultados de } 4 \text { estudos incluídos mostram } \\
\text { consistentemente piora significativa }(p<0,05) \text { do sangramento na } \\
\text { sondagem }(4,21 \% \text { (IC 95\%: } 0,32,8,11)) \text {, perda de inserção clínica }(0,16 \\
\text { mm (IC 95\%: } 0,05,0,27)) \text {, profundidade da bolsa periodontal (PPD) } \\
(0,14 \mathrm{~mm}(95 \% \text { CI: } 0,06,0,23)) \text { e porcentagem de PPD } 4-5 \mathrm{mm:} 1,72 \% \\
(95 \% \text { CI: } 0,11,3,34) 6 \text { meses após BS, mas nenhuma mudança após } 12 \\
\text { meses. }\end{array}$ \\
\hline
\end{tabular}

porcentagem de locais de sangramento (MD: -0,21; IC95\% - 0,77 a 0,35), e profundidade de sondagem de bolso (MD: 0,08 CI95\% -0,14 a $0,31)$ não foram diferentes antes e após a cirurgia bariátrica. No entanto, o índice de placa foi menor após do que antes da cirurgia bariátrica (MD: -1,29; IC 95\% -2,34 a -0,24).

Fonte: Acervo dos autores (2021).

\section{Discussão}

Dentro desse trabalho, foram analisados 17 artigos, sendo 8 revisões de literatura, 8 estudos e 1 pesquisa, e observouse a presença da doença periodontal em obesos e em indivíduos submetidos a cirurgia bariátrica, suas causas e como essa doença é manifestada nesses dois grandes grupos, abordando a temática do trabalho. As provas que mostram a evolução da 
doença periodontal após a cirurgia bariátrica apoiam que é preciso mais estudos de intervenção, que até agora têm mostrado bons resultados.

O estudo de Franco et al. (2020) mostraram que estar em sobrepeso ou com obesidade está associado com um maior risco de periodontite. O tecido adiposo presente nos obesos é um órgão endócrino que libera várias citocinas pró-inflamatórias, possíveis de modificar a resposta dos tecidos periodontais ao ambiente bucal. O trabalho de Pataro et al. (2016) traz que a cirurgia bariátrica é uma forma de reduzir esse tecido adiposo em obesos, sendo cada um com seus critérios específicos.

Logo Levine (2012) contradiz, trazendo que não é estabelecido uma causa diretamente ligada entre a obesidade e a doença periodontal. O que deve ser declarado com alguma confiança é que a obesidade é um dos fatores dessa complexa e multifatorial relação, exigindo uma investigação com conclusões mais detalhadas.

A cirurgia bariátrica pode ser realizada em pacientes obesos com doença periodontal, em tratamento e estabilizados. Em seu artigo, Dos santos et al. (2019) fizeram uma análise de todos os estudos incluídos em seu trabalho e observaram uma elevada heterogeneidade, devido a isso é fundamental uma padronização global dos estudos periodontais com a utilização de índices e parâmetro de avaliação de cada grupo em especifico.

A saúde bucal deficiente é frequentemente encontrada em obesos, nos resultados do estudo feito por Bastos et al. (2018), foram relatados pelos pacientes a falta da utilização do fio dental, sendo um fator de risco para a doença periodontal, porque leva ao acumulo de placa bacteriana, consequentemente ocasionando no sangramento gengival, sendo portanto a gengivite uma inflamação do periodonto de proteção, que está associada a obesidade.

O estudo realizado por Moura Grec et al. (2014), trouxeram que a hipossalivação é comum entre os pacientes bariátricos, pois devido ao estômago reduzido, existe uma dificuldade em beber água, tendo como consequência a boca seca também conhecida como xerostomia, ocasionando diversos problemas bucais, dentre eles a doença periodontal, aumento da cárie dentaria e halitose. Mais da metade dos pacientes bariátricos avaliados por Schwenger et al. (2020), tinham hipossalivação antes da cirurgia, tendo o seu fluxo salivar melhorado após realizar o procedimento, pode haver outras razões para a persistência da xerostomia, que incluem aumento de refluxos, vômitos e associações com outras condições sistêmicas.

Os estudos observados por Colak et al. (2021) perceberam um agravamento no prazo de 6 meses do estado periodontal depois da cirurgia bariátrica, apresentou uma melhora após 1 ano desde o início da cirurgia, que corresponde ao período de adaptação alimentar e pós-operatório crítico. Além destes autores, Dos santos et al. (2019) concluiram que o acompanhamento de períodos mais longos parece ser necessário para medir as alterações clínicas periodontais e para permitir que o corpo reaja ao impacto cirúrgico e atinja um estado de equilíbrio.

Durante a discussão de seu artigo, De souza et al. (2018) trouxeram na análise da sua revisão, que a cirurgia bariátrica pode melhorar o estado periodontal do paciente com obesidade, dando ênfase no índice de placa, que foi menor após do que antes da cirurgia. Sendo assim, Porcelli et al. (2019) afirmaram que esses pacientes bariátricos precisam estar inseridos em um programa de promoção de saúde bucal com acompanhamento odontológico do período anterior e posterior a cirurgia, a fim de prevenir o desenvolvimento de doenças bucais, trazendo ganhos para qualidade de vida e bem-estar dos pacientes bariátricos.

Neste outro estudo, realizado em obesos que perderam peso após realizarem a cirurgia bariátrica e os que não realizaram, Sales-Peres et al. (2017), trouxeram que foi possível observar inflamação gengival com sangramento em alguns sítios, em contrapartida aqueles que não tiveram o seu peso reduzido, foi perceptível apenas pequenas alterações na bolsa periodontal e ganho de profundidade. Já Marsicano et al. (2012) observaram uma condição mais prevalecente de bolsas periodontais em pacientes bariátricos. Portanto a relação entre perda de peso após cirurgia e doença periodontal devem ser mais exploradas por futuros estudos prospectivos.

Em seu estudo clinico exploratório, Lakkis et al. (2012) mostraram uma melhora na resposta à terapia periodontal não cirúrgica em pacientes obesos que perderam peso significativo após a cirurgia bariátrica em comparação aqueles pacientes 
obesos que não foram submetidos a tal cirurgia. Estudos longitudinais adicionais com amostra maior são necessários para confirmar ou refutar os resultados do presente estudo.

A má absorção de nutrientes pode induzir alterações metabólicas, tais como reabsorção e perda óssea, e por isso Moura- grec et al. (2012), trouxeram em seu trabalho um relato de caso que foi realizado, tomografias computadorizadas 1 e 2 anos após cirurgia bariátrica para medir a perda óssea e a diferença entre esses dois períodos foi de 0,93 $\mathrm{mm}$, sendo maior 2 anos após a cirurgia bariátrica. Os locais com maior profundidade de sondagem foram confirmados como os áreas de perda óssea, assim exercendo um impacto negativo sobre o estado periodontal.

Segundo Pataro et al. (2012), recomendam-se que os indivíduos bariátricos, após a cirurgia, adquiram novos hábitos fisiológicos e comportamentais para estabelecer um novo estilo de vida, assumindo condutas saudáveis na sua rotina, incluindo a higiene oral, que estão intrinsicamente relacionados com a saúde do periodonto. Assim como Dos santos et al. (2019) defendem que a multidisciplinaridade no acompanhamento do período pré-operatório e pós-operatório pode minimizar estes efeitos e melhorar a saúde oral.

É necessário que os médicos aconselhem os pacientes que irão realizar a cirurgia bariátrica se submeterem a uma avaliação periodontal antes, assim como seu seguimento após a cirurgia bariátrica, Fontanille et al. (2018) trazem que devido as características disabsortivas da cirurgia que favorecem a não absorção de nutrientes como vitaminas B12, D, cálcio, ferro e ácido fólico, que são vitais para manutenção da saúde periodontal, é recomendado o monitoramento nutricional, além da suplementação de vitaminas e minerais. Moura Grec et al. (2012) afirmam que essas medidas são essenciais para a prevenção de perda óssea e tratamento das consequências metabólicas advindas da cirurgia bariátrica, incluindo suas possíveis repercussões na saúde bucal.

\section{Conclusão}

Diante do exposto, percebe-se a necessidade de mais estudos envolvendo os grupos citados, pois foi observada a falta da determinação de um protocolo para oferecer um melhor tratamento a cada caso, levando em conta qualquer particularidade aparente. A abordagem de um cirurgião-dentista para realizar a avaliação periodontal, prevenção, tratamento e controle tanto antes da cirurgia como durante e no pós-operatório é de grande importância para manutenção da saúde bucal durante todo o processo de emagrecimento. A equipe que cuida de pacientes submetidos à cirurgia de bariátrica deve ser multidisciplinar para que esses cuidados sejam feitos de maneira adequada e completa, a fim de monitorar o seu estado periodontal ao longo de todo processo.

Trabalhos futuros com abordagens em tentar definir um protocolo de higiene oral para obter resultados mais eficientes podem ser realizados para fornecer um norte aos profissionais nos cuidados odontológicos de pacientes obesos e pacientes obesos submetidos a cirurgia bariátrica. A valorização do cirurgião-Dentista na equipe multidisciplinar é muito importante para a melhoria da qualidade de vida nesse âmbito.

\section{Referências}

Bastos, I., Alves, E. S., Sousa, C. D., Martins, G. B., Campos, E. J., \& Daltro, C. (2018). Prevalence of risk factors for oral diseases in obese patients referred for bariatric surgery. Journal of the American Dental Association (1939), 149(12), 1032-1037. https://doi.org/10.1016/j.adaj.2018.07.024

Čolak, D., Gašperšič, R., Kučič, A. C., Pintar, T., \& Gašpirc, B. (2021). The effect of bariatric surgery on periodontal health: systematic review and metaanalyses. Archives of medical science : AMS, 17(4), 1118-1127. https://doi.org/10.5114/aoms/135880

de Moura-Grec, P. G., Marsicano, J. A., Rodrigues, L. M., \& de Carvalho Sales-Peres, S. H. (2012). Alveolar bone loss and periodontal status in a bariatric patient: a brief review and case report. European journal of gastroenterology \& hepatology, 24(1), 84-89. https://doi.org/10.1097/MEG.0b013e32834bebb3

de Moura-Grec, P. G., Yamashita, J. M., Marsicano, J. A., Ceneviva, R., de Souza Leite, C. V., de Brito, G. B., Brienze, S. L., \& de Carvalho Sales-Peres, S. H. (2014). Impact of bariatric surgery on oral health conditions: 6-months cohort study. International dental journal,64(3), 144-149. https://doi.org/10.1111/idj.12090 
Dos Santos, M., Pellizzer, E. P., SoutoMaior, J. R., Casado, B., Luna Gomes, J. M., Vasconcelos, B., \& Moraes, S. (2019). Clinical periodontal conditions in individuals after bariatric surgery: a systematic review and meta-analysis. Surgery for obesity and related diseases : official journal of the American Society for Bariatric Surgery, 15(10), 1850-1859. https://doi.org/10.1016/j.soard.2019.07.008

Estrela, C. (2018). Metodologia Científica: Ciência, Ensino, Pesquisa. Editora Artes Médicas.

Fontanille, I., Boillot, A., Rangé, H., Carra, MC, Sales-Peres, S., Czernichow, S., \& Bouchard, P. (2018). Cirurgia bariátrica e estado periodontal: uma revisão sistemática com meta-análise. Cirurgia para obesidade e doenças relacionadas: jornal oficial da Sociedade Americana de Cirurgia Bariátrica, 14 (10), 16181631. https://doi.org/10.1016/j.soard.2018.07.017

Franco, R., Barlattani, A., Jr, Perrone, MA, Basili, M., Miranda, M., Costacurta, M., Gualtieri, P., Pujia, A., Merra, G., \& Bollero, P. (2020). Obesidade, cirurgia bariátrica e doença periodontal: uma atualização da literatura. Revisão europeia para ciências médicas e farmacológicas, 24 (9), 50365045. https://doi.org/10.26355/eurrev_202005_21196

Girano Castaños, J., \& Robello Malatto, J. (2020). Relación entre obesidad y enfermedad periodontal: revisión de la literatura. Horizonte Médico (Lima), 20(3).

Kinane, D. F., Stathopoulou, P. G., \& Papapanou, P. N. (2017). Periodontal diseases. Nature Reviews Disease Primers, $3(1)$, 1-14.

Lakkis, D., Bissada, N. F., Saber, A., Khaitan, L., Palomo, L., Narendran, S., \& Al-Zahrani, M. S. (2012). Response to periodontal therapy in patients who had weight loss after bariatric surgery and obese counterparts: a pilot study. Journal of periodontology, 83(6), 684-689. https://doi.org/10.1902/jop.2011.110230

Levine R. (2012). Obesity and oral disease - a challenge for dentistry. British dental journal, 213(9), 453-456. https://doi.org/10.1038/sj.bdj.2012.1009

Maria de Souza, G., Willya Douglas de Oliveira, D., Santos Lages, F., Andrade Fernandes, I., \& Gabriel Moreira Falci, S. (2018). Relationship between bariatric surgery and periodontal status: a systematic review and meta-analysis. Surgery for obesity and related diseases: official journal of the American Society for Bariatric Surgery, 14(8), 1205-1216. https://doi.org/10.1016/j.soard.2018.04.018

Marsicano, J. A., Sales-Peres, A., Ceneviva, R., \& de C Sales-Peres, S. H. (2012). Evaluation of oral health status and salivary flow rate in obese patients after bariatric surgery. European journal of dentistry, 6(2), 191-197.

Moura-Grec, P. G., Assis, V. H., Cannabrava, V. P., Vieira, V. M., Siqueira, T. L., Anaguizawa, W. H., \& Sales-Peres, S. H. (2012). Systemic consequences of bariatric surgery and its repercussions on oral health. Arquivos brasileiros de cirurgia digestiva: $A B C D=B r a z i l i a n$ archives of digestive surgery, $25(3)$, 173-177. https://doi.org/10.1590/s0102-67202012000300008

Mur Villar, N., García San Juan, C., Castellanos González, M., Sexto Delgado, N., Méndez Castellanos, C., \& Gamio Pruna, W. (2017). La influencia de la obesidad y la ateroesclerosis en la etiología y patogenia de las enfermedades periodontales. Medisur, 15(1), 93-106.

Nascimento, G. G., Peres, K. G., Mittinty, M. N., Mejia, G. C., Silva, D. A., Gonzalez-Chica, D., \& Peres, M. A. (2017). Obesity and periodontal outcomes: a population-based cohort study in Brazil. Journal of periodontology, 88(1), 50-58.

Pataro, A. L., Cortelli, S. C., Abreu, M. H., Cortelli, J. R., Franco, G. C., Aquino, D. R., Cota, L. O., \& Costa, F. O. (2016). Frequency of periodontal pathogens and Helicobacter pylori in the mouths and stomachs of obese individuals submitted to bariatric surgery: a cross-sectional study. Journal of applied oral science: revista FOB, 24(3), 229-238. https://doi.org/10.1590/1678-775720150534

Pataro, A. L, Costa, F. O, Cortelli, S. C, Cortelli, J. R, Dupim Souza, A. C, Nogueira Guimarães Abreu, M. .H, Girundi, M. G, \& Costa, J. E (2012). Influência da obesidade e da cirurgia bariátrica na condição periodontal. Journal of periodontology, 83 (3), 257-266. https://doi.org/10.1902/jop.2011.100782

Porcelli, I., Corsi, N. M., Fracasso, M., Pascotto, R. C., Cardelli, A., Poli-Frederico, R. C., Nasser, D., \& Maciel, S. M. (2019). oral health promotion in patients with morbid obesity after gastroplasty: a randomized clinical trial. Arquivos brasileiros de cirurgia digestiva: ABCD = Brazilian archives of digestive surgery, 32(2), e1437. https://doi.org/10.1590/0102-672020190001e1437

Prado, r. 1. d., santos, n. d. m., silva, k. e. d., rodrigues, m. c., taguti, j. y. d. t., \& marsicano, j. a. (2020). Oral conditions and the impact on quality of life of morbidly obese and bariatric patients. RGO-Revista Gaúcha de Odontologia, 68.

Sales-Peres, S., Sales-Peres, M. C., Ceneviva, R., \& Bernabé, E. (2017). Weight loss after bariatric surgery and periodontal changes: a 12-month prospective study. Surgery for obesity and related diseases official journal of the American Society for Bariatric Surgery, 13(4), 637-642. https://doi.org/10.1016/j.soard.2016.08.007

Schwenger, K., Alghamdi, M. M., Ghorbani, Y., Jackson, T. D., Okrainec, A., \& Allard, J. P. (2020). Hyposalivation is prevalent in bariatric patients but improves after surgery. Surgery for obesity and related diseases: official journal of the American Society for Bariatric Surgery, 16(10), 1407-1413. https://doi.org/10.1016/j.soard.2020.06.005

Teixeira, F. C. F., Marin-Leon, L., Gomes, E. P., PedrÃo, A. M. N., Pereira, A. D. C., \& Francisco, P. M. S. B. (2020). Relationship between periodontitis and subclinical risk indicators for chronic non-communicable diseases. Brazilian Oral Research, 34.

Yamashita, Y., \& Takeshita, T. (2017). The oral microbiome and human health. Journal of oral science, 59(2), 201-206. 